\title{
Massive Yang-Mills Theory in Abelian Gauges
}

\author{
Ulrich ELLWANGER ${ }^{\mathrm{a}}$ 冈, Nicolás WSCHEBOR ${ }^{\mathrm{a}, \mathrm{b}}$ \\ a Laboratoire de Physique Théorique f \\ Université de Paris XI, Bâtiment 210, F-91405 ORSAY Cedex, France \\ b Institutos de Física \\ Facultad de Ciencias (Calle Iguá 4225, esq. Mataojo) \\ and Facultad de Ingeniería (C.C.30, CP 1100), Montevideo, Uruguay
}

\begin{abstract}
We prove the perturbative renormalisability of pure $\mathrm{SU}(2)$ Yang-Mills theory in the abelian gauge supplemented with mass terms. Whereas mass terms for the gauge fields charged under the diagonal U(1) allow to preserve the standard form of the Slavnov-Taylor identities (but with modified BRST variations), mass terms for the diagonal gauge fields require the study of modified Slavnov-Taylor identities. We comment on the renormalization group equations, which describe the variation of the effective action with the different masses. Finite renormalized masses for the charged gauge fields, in the limit of vanishing bare mass terms, are possible provided a certain combination of wave function renormalization constants vanishes sufficiently rapidly in the infrared limit.
\end{abstract}

PACs: 11.15.-q, 11.15.Pg, 12.38.Aw

LPT Orsay 02-39

April 2002

\footnotetext{
*Email: ellwanger@th.u-psud.fr

${ }^{\dagger}$ Email: wschebor@th.u-psud.fr

‡Unité Mixte de Recherche - CNRS - UMR 8627
} 


\section{Introduction}

Nowadays it is generally accepted that massive Yang-Mills theory, in its perturbative formulation, requires a Higgs field in order to avoid a clash between renormalizability and (perturbative) unitarity [1]. Nevertheless the study of massive pure Yang-Mills theories is important for several reasons: First, perturbative calculations of many physically interesting quantities are plagued by infrared divergences, which require an infrared regularization. Such an infrared regularization should be consistent, i.e. preserve renormalizability and allow to recover perturbative unitarity in the limit where it is removed (as long as one remains within a perturbative framework like partonic matrix elements). Since mass terms belong to the simplest infrared regulators, one should master their corresponding properties. Second, lattice results on the behaviour of gauge field propagators in the infrared regime indicate that nonperturbative effects induce a massive behaviour of these propagators [2, 3].

The advantage of Abelian gauges [4, 5n in the infrared regime of Yang-Mills theories is that they allow to formulate most clearly the monopole condensation picture of confinement [6]. It is believed that the confining phase of a Yang-Mills theory is dual to a Landau-Ginzburg or Higgs phase, in which the off-diagonal gluons would be massive. Indeed results of lattice simulations in abelian gauges [3] indicate that here the propagators of the "off-diagonal" gauge fields (associated with off-diagonal generators) and "diagonal" gauge fields (associated with diagonal $U(1)$ subgroups) behave very differently: The massive behaviour is observed only for the off-diagonal gauge fields [3]. Some arguments which serve to explain this phenomenon have been proposed in [7, 8]. The diagonal gauge fields (including their monopole configurations) may then be responsable for confinement, perhaps in some analogy to compact electrodynamics [9]. Moreover it has recently been shown that some generic families of abelian gauge theories confine [10]. We can then expect that one of these latter models represents the long distance behavior of the Yang-Mills theory, at energy scales below the masses of the off-diagonal gauge fields.

In addition it should be noted that the non-perturbative existence of an abelian gauge fixing for $(\mathrm{SU}(2))$ Yang-Mills theory, including the solution of the Gribov problem [11, has been proved for a covariant, local and BRST-invariant action [12].

For the above reasons the study of massive Yang-Mills theory in abelian gauges is required. As in the case of various versions of massive Yang-Mills theories in gauges preserving the global part of the gauge group [13, 14] one should be able to define a renormalizable gauge fixing sector of the theory. This is the main task of the present paper. As usual, to this end one has to study slightly modified versions of SlavnovTaylor identities and some additional symmetries of the action, which should be preserved by the ultra-violet regularisation (as dimensional regularisation).

Since we insist on a complete infrared regularisation of the theory we study the effects of mass terms for both the off-diagonal and diagonal gauge fields (which 
do not, however, have to be the same). The required modifications of the SlavnovTaylor identities turn out to be quite different: Whereas mass terms for the diagonal gauge fields require the introduction of additional sources which couple to its BRST variation, mass terms for the off-diagonal gauge fields (accompanied by suitable mass terms for the off-diagonal ghosts) alone can be coped with by slight variations of the BRST transformations or the Slavnov-Taylor identities.

Once renormalisability of the theory has been shown, one can proceed and discuss particularly convenient renormalisation schemes. Since the theory is, by construction, infrared save, one can renormalise at vanishing external momenta. The (asymptotic) scaling behaviour of the Green functions can be described by the Callan-Symanzik (CS) equation [15] which, strictly speaking, describes the variation of the Green functions with the masses.

The CS equation plays a role somewhat in between the usual renormalisation group equations in some mass independent substraction scheme (where the first two terms of the $\beta$-functions are universal) and the Exact Renormalisation Group (ERG) Equations [16, 17], which are based on general momentum dependent cutoff terms (quadratic in the fields) in the action. Since also here the mass terms can play the role of an infrared regulator (whose variation is described by the ERGs), this issue will be discussed briefly below.

Given the lattice results on the massive behaviour of propagators of the offdiagonal gauge fields it is natural to ask whether it is possible to a) remove the mass terms as explicit infrared regulators, b) maintain finite mass terms for the off-diagonal gauge fields in the effective action, but c) recover the standard SlavnovTaylor identities without additional terms. We find, within a parametrization of the effective action by its perturbatively relevant terms only, that this situation is possible. Of course, a sufficiently violent (divergent) infrared behaviour of the wave function renormalization constants is required for this purpose. Most interestingly the Slavnov-Taylor identities imply that, amongst others, it is the wave function renormalization constant of the diagonal gauge fields (which are supposedly responsable for confinement and vanishing in the infrared [18]), which appears in the denominator of the renormalised mass term for the off-diagonal gauge fields and could thus render it non-vanishing even in the limit of vanishing bare mass term. Of course we cannot prove this behaviour here. Although this scenario would allow for standard Slavnov-Taylor identities together with massive off-diagonal gauge fields, this is not sufficient for perturbative unitarity. On the other hand, perturbative unitarity in a confining theory is possibly not even required; thus we will not discuss perturbative unitarity any further in the rest of the paper.

The paper is organized as follows. First, in section 2, we introduce the model (massive Yang-Mills theory in an abelian gauge) and its symmetries. In section 3 we prove its renormalisability. For simplicity, the proofs are presented for $\mathrm{SU}(2)$, but the generalisation to $\mathrm{SU}(\mathrm{N})$ or the inclusion of gauge invariant matter is straightforward. The massless limit is smooth and the usual Slavnov-Taylor identities are recovered in this limit. In section 4 we study first the CS equations. We discuss some properties 
of the $\beta$ functions and anomalous dimensions, and the different roles of the masses for the diagonal and off-diagonal gluons. We comment the relation with the ERG equations in this respect, and finish with a speculation on the possibility to obtain massive off-diagonal gluons in the confining phase. Conclusions are drawn in section 5.

\section{The Model}

We consider pure $S U(2)$ Euclidean Yang-Mills theory. $A_{\mu}$ denote the gauge fields associated to the $U(1)$ subgroup, and $W_{\mu}^{ \pm}$are the off-diagonal gauge fields with the corresponding $U(1)$ charges. The classical action $S$ reads

$$
S=\int d^{4} x\left\{\mathcal{L}_{Y M}+\mathcal{L}_{G F}+\mathcal{L}_{m}\right\}
$$

where $\mathcal{L}_{Y M}$ is the Yang-Mills Lagrangian

$$
\begin{aligned}
\mathcal{L}_{Y M}= & \frac{1}{4}\left(\partial_{\mu} A_{\nu}-\partial_{\nu} A_{\mu}\right)^{2}+\frac{i g}{2}\left(\partial_{\mu} A_{\nu}-\partial_{\nu} A_{\mu}\right)\left(W_{\mu}^{+} W_{\nu}^{-}-W_{\mu}^{-} W_{\nu}^{+}\right) \\
& -\frac{g^{2}}{4}\left(W_{\mu}^{+} W_{\nu}^{-}-W_{\mu}^{-} W_{\nu}^{+}\right)^{2}+\frac{1}{2}\left(D_{\mu} W_{\nu}^{+}-D_{\nu} W_{\mu}^{+}\right)\left(D_{\mu} W_{\nu}^{-}-D_{\nu} W_{\mu}^{-}\right),
\end{aligned}
$$

and $\mathcal{L}_{G F}$ is the gauge fixing part

$$
\begin{aligned}
\mathcal{L}_{G F}= & -\frac{\beta}{2} \phi_{3}^{2}-\phi_{3} \partial_{\mu} A_{\mu}+\partial_{\mu} \bar{c}^{3}\left(\partial_{\mu} c^{3}+i g\left(W_{\mu}^{+} c^{-}-W_{\mu}^{-} c^{+}\right)\right) \\
& -\alpha\left(\phi^{+}-i g c^{3} \bar{c}^{+}\right)\left(\phi^{-}+i g c^{3} \bar{c}^{-}\right) \\
& -\left(\phi^{+}-i g c^{3} \bar{c}^{+}\right) D_{\mu} W_{\mu}^{-}-\left(\phi^{-}+i g c^{3} \bar{c}^{-}\right) D_{\mu} W_{\mu}^{+}+D_{\mu} \bar{c}^{+} D_{\mu} c^{-}+D_{\mu} \bar{c}^{-} D_{\mu} c^{+} \\
& +g^{2}\left(W_{\mu}^{+} \bar{c}^{-}-W_{\mu}^{-} \bar{c}^{+}\right)\left(W_{\mu}^{+} c^{-}-W_{\mu}^{-} c^{+}\right)-g^{2} \alpha \bar{c}^{+} c^{-} \bar{c}^{-} c^{+}
\end{aligned}
$$

The derivatives $D_{\mu}$ are $U(1)$ covariant. For convenience we write the masses for the gauge bosons $W_{\mu}^{ \pm}, A_{\mu}$, charged and neutral ghosts in the form $m^{2}, \lambda m^{2}, \chi_{c} m^{2}$ and $\chi_{3} m^{2}$, respectively:

$$
\mathcal{L}_{m}=m^{2}\left(W_{\mu}^{+} W_{\mu}^{-}+\chi_{c}\left(\bar{c}^{+} c^{-}+\bar{c}^{-} c^{+}\right)+\frac{\lambda}{2} A_{\mu} A_{\mu}+\chi_{3} \bar{c}^{3} c^{3}\right)
$$

In the massless case, renormalisability for a two parameter family of abelian gauges has been shown in [19]. Our choice for $\mathcal{L}_{G F}$ corresponds to a particular

choice for the parameters in [19], which has also been studied and proven to be 
stable under renormalization in [20]. The techniques used are the usual BRST [21] symmetry and some additional identities exposed below.

$\mathcal{L}_{G F}$ is invariant under the (nilpotent) BRST transformations

$$
\begin{array}{ll}
s A_{\mu}=\partial_{\mu} c^{3} \pm i g W_{\mu}^{ \pm} c^{\mp}, & s W_{\mu}^{ \pm}=D_{\mu} c^{ \pm} \mp i g c^{3} W_{\mu}^{ \pm}, \\
s c^{3}=-i g c^{+} c^{-}, & s c^{ \pm}=\mp i g c^{3} c^{ \pm}, \\
s \bar{c}^{3}=-\phi_{3}, & s \bar{c}^{ \pm}=-\phi^{ \pm}, \\
s \phi_{3}=0, & s \phi^{ \pm}=0 .
\end{array}
$$

Here $s$ denotes, as usual, the operator generating BRST transformations:

$$
\delta \varphi=\xi s \varphi
$$

where $\xi$ is a Grassmann parameter. (Here and below $\varphi$ denote all possible fields; whenever necessary, we indicate explicitely its $U(1)$ charge \pm 1 .) We introduce the usual sources for composite operators for the BRST variations and, in addition, a source $\rho$ for the variation of $\mathcal{L}_{m}$ :

$$
\mathcal{L} \longrightarrow \mathcal{L}-K_{\mu}^{3} s A_{\mu}-K_{\mu}^{ \pm} s W_{\mu}^{\mp}-L^{3} s c^{3}-L^{ \pm} s c^{\mp}-\rho \Sigma_{\rho}
$$

with

$$
\begin{aligned}
\Sigma_{\rho}= & s\left(W_{\mu}^{+} W_{\mu}^{-}+\chi_{c}\left(\bar{c}^{+} c^{-}+\bar{c}^{-} c^{+}\right)+\frac{\lambda}{2} A_{\mu} A_{\mu}+\chi_{3} \bar{c}^{3} c^{3}\right) \\
= & W_{\mu}^{\mp} D_{\mu} c^{ \pm}-\chi_{c}\left(\phi^{ \pm} \mp i g c^{3} \bar{c}^{ \pm}\right) c^{\mp}+\lambda A_{\mu}\left(\partial_{\mu} c^{3} \pm i g W_{\mu}^{ \pm} c^{\mp}\right) \\
& +\chi_{3}\left(i g \bar{c}^{3} c^{+} c^{-}-\phi_{3} c^{3}\right)
\end{aligned}
$$

Now the mass terms lead to a relatively simple modification of the Slavnov-Taylor identity for the action, which becomes

$$
\begin{aligned}
& \int d^{4} x\left\{\frac{\delta \Gamma}{\delta K_{\mu}^{3}} \frac{\delta \Gamma}{\delta A_{\mu}}+\frac{\delta \Gamma}{\delta K_{\mu}^{ \pm}} \frac{\delta \Gamma}{\delta W_{\mu}^{\mp}}+\frac{\delta \Gamma}{\delta L^{3}} \frac{\delta \Gamma}{\delta c^{3}}+\frac{\delta \Gamma}{\delta L^{ \pm}} \frac{\delta \Gamma}{\delta c^{\mp}}-\phi_{3} \frac{\delta \Gamma}{\delta \bar{c}^{3}}-\phi^{ \pm} \frac{\delta \Gamma}{\delta \bar{c}^{\mp}}\right\} \\
& +m^{2} \frac{\partial \Gamma}{\partial \rho}=0 .
\end{aligned}
$$

In addition to the non-linear Slavnov-Taylor identity (2.9) the action has the following linearly realised symmetries:

a) ghost number conservation induced by $\delta c=\epsilon c, \delta \bar{c}=-\epsilon \bar{c}$,

b) charge conjugation $\varphi^{3} \rightarrow-\varphi^{3}, \varphi^{ \pm} \rightarrow \varphi^{\mp}$,

c) invariance under a constant shift of the neutral antighost, which is only broken by the neutral ghost mass and a term proportional to $\rho$, which can be written as a variation with respect to $L^{3}$. It gives rise to the identity 


$$
\frac{\delta \Gamma}{\delta \bar{c}^{3}}-\partial_{\mu} \frac{\delta \Gamma}{\delta K_{\mu}^{3}}-m^{2} \chi_{3} c^{3}-\chi_{3} \rho \frac{\delta \Gamma}{\delta L^{3}}=0 .
$$

d) the U(1) gauge symmetry is only broken by the gauge-fixing term $\phi_{3} \partial_{\mu} A_{\mu}$, the $A_{\mu}$ mass term and a term proportional to $\rho$, which can be expressed in terms of a variation with respect to $K_{\mu}^{3}$. An infinitesimal $U(1)$ transformation allows to derive the following Ward identitiy:

$$
\partial_{\mu} \frac{\delta \Gamma}{\delta A_{\mu}} \pm i g \varphi^{ \pm} \frac{\delta \Gamma}{\delta \varphi^{ \pm}}-\partial^{2} \phi_{3}-\rho \partial_{\mu} \frac{\delta \Gamma}{\delta K_{\mu}^{3}}-m^{2} \lambda \partial_{\mu} A_{\mu}=0
$$

e) the Grassmannian U(1) gauge symmetry

$$
\delta c^{3}(x)=\eta(x), \delta \phi^{ \pm}(x)= \pm i g \eta(x) \bar{c}^{ \pm}(x)
$$

is only broken by the neutral ghost kinetic term and terms proportional to the sources $K, L$ and $\rho$, which gives raise to the identity:

$$
\begin{aligned}
\frac{\delta \Gamma}{\delta c^{3}} \pm i g \bar{c}^{ \pm} \frac{\delta \Gamma}{\delta \phi^{ \pm}}= & \left(\partial^{2}-m^{2} \chi_{3}\right) \bar{c}^{3}-\partial_{\mu} K_{\mu}^{3}+i g\left( \pm K_{\mu}^{ \pm} W_{\mu}^{\mp} \mp L^{ \pm} c^{\mp}\right) \\
& -\rho \lambda \partial_{\mu} A_{\mu}-\rho \chi_{3} \phi_{3} .
\end{aligned}
$$

In [20] this equation (for $\lambda=\chi_{3}=0$ ) has been denoted "neutral ghost field equation". It implies that the charged fields $\phi^{ \pm}$and the neutral ghosts appear in the effective action only in the combination $\phi^{ \pm} \mp i g c^{3} \bar{c}^{ \pm}$, apart from the terms linear in the other fields which will thus remain unrenormalised.

\section{Proof of perturbative renormalisability}

The proof of perturbative renormalisability requires an ultraviolet regulator (like dimensional regularisation) which respects all the above symmetries or identities, and goes by induction in the number of loops. Using usual power-counting arguments, new infinities to loop order $n$ can appear only proportional to terms in the effective action $\Gamma$ with dimension less or equal than four, once all infinities to all loop orders less than $n$ have been reabsorbed into redefinitions of couplings and fields. One has to show that all possible new divergencies can be reabsorbed into redefinitions of the fields, sources and parameters already present.

First we note that no divergencies quadratic in the sources $K, L$ and $\rho$ are possible. The following facts are required to this end: $K, L$ and $\rho$ have mass dimensions 2, 2 and 1, respectively. $K$ and $\rho$ anticommute and have ghost number -1 . The sources $L$ are bosonic and have ghost number -2 . Then ghost number 
conservation implies that no terms of dimension four or less exist with two or more sources $K$ and/or $L$, since one would need too many additional ghost fields in order to end up with ghost number 0 . The same argument forbid terms $\sim K \cdot L$ and $\sim K \cdot \rho$. In addition, because $\rho$ anticommutes, terms without derivatives $\sim \rho^{2}$ vanish. Hence we cannot construct terms quadratic in any sources with vanishing ghost number and dimension less or equal than four.

The (divergent) contributions to $\Gamma$ linear in the sources $K_{\mu}^{ \pm}, K_{\mu}^{3}, L^{ \pm}$and $L^{3}$, to loop order $n$, modify the effective BRST variations of the fields $W_{\mu}^{ \pm}, A_{\mu}, c^{ \pm}$and $c^{3}$ to loop order $n$. They are strongly constraint by the Slavnov-Taylor identity (2.9), and the constraints a) - e) on the effective action. Let us denote the effective BRST variations to loop order $n$ by

$$
s_{n} \varphi=s \varphi+\Delta \widetilde{s}_{n} \varphi
$$

where $s \varphi$ is of the classical form (2.3) by assumption. $\Delta \widetilde{s}_{n} \varphi$ is the divergent contribution from loop order $n$, which can be obtained from the coefficient of the corresponding source terms. We have $\Delta \widetilde{s}_{n} \bar{c}=0$ (since, from $(2.9), s_{n} \bar{c}=s \bar{c}=-\phi$ to all loop orders) and $\Delta \widetilde{s}_{n} \phi=0$ (since, from (2.9), $s_{n} \phi=s \phi=0$ to all loop orders). The terms linear in $K$ and $L$ in the Slavnov-Taylor identity (2.9) (where the term $\sim m^{2}$ does not contribute) to order $\Delta$ imply

$$
s_{n}^{2} \varphi=0+\mathcal{O}\left(\Delta^{2}\right)
$$

on the fields $A_{\mu} W_{\mu}$ and $c$, which means that the quantum corrected BRST variations remain nilpotent. Taking the constraints a) - e) on the effective action into account, the most general form of the BRST variations to order $n$ is then

$$
\begin{array}{ll}
s_{n} A_{\mu}=\partial_{\mu} c^{3}+i g \sqrt{\frac{Z_{W} Z_{c} Y_{3}}{Z}}\left(W_{\mu}^{+} c^{-}-W_{\mu}^{-} c^{+}\right), & s_{n} c^{3}=-i g Y_{3} Z_{c} c^{+} c^{-}, \\
s_{n} W_{\mu}^{ \pm}=\sqrt{\frac{Z Y_{3} Z_{c}}{Z_{W}}} D_{\mu} c^{ \pm} \mp i g c^{3} W_{\mu}^{ \pm}, & s_{n} c^{ \pm}=\mp i g c^{3} c^{ \pm}, \\
s_{n} \bar{c}^{3}=-\phi_{3}, & s_{n} \bar{c}^{ \pm}=-\phi^{ \pm}, \\
s_{n} \phi_{3}=0, & s_{n} \phi^{ \pm}=0 .
\end{array}
$$

The divergent constants $Z, Z_{W}$, and $Z_{c}$ will play the role of the wave function renormalization constants of the fields $A_{\mu}, W_{\mu}$ and $c^{ \pm}$, and $\sqrt{Y_{3}}$ resp. $\sqrt{Y_{3}^{-1}}$ will renormalize the neutral ghosts $c^{3}$ resp. $\bar{c}^{3}$. The non-renormalization of the terms $\sim c^{3}$ in the variations has its origin in the identity (2.13).

Next we consider the quantum corrections to $\Sigma_{\rho}$, the BRST variation of the mass term. Let us denote it by

$$
\Sigma_{n, \rho}=\Sigma_{\rho}+\Delta \widetilde{\Sigma}_{n, \rho}
$$

where the first term on the right hand side is of the classical form (2.8) by assumption, and $\Delta \widetilde{\Sigma}_{n, \rho}$ is the divergent contribution from loop order $n$. 
The terms of order $\rho$ in the Slavnov-Taylor identity (2.9) now imply (again the term $\sim m^{2}$ does not contribute)

$$
s_{n} \Sigma_{n, \rho}=0+\mathcal{O}\left(\Delta^{2}\right) .
$$

Together with the constraints a) - e) $\Sigma_{n, \rho}$ is then necessarily of the form

$$
\begin{aligned}
& \Sigma_{n, \rho}=\lambda A_{\mu}\left(\partial_{\mu} c^{3}+i g \sqrt{\frac{Y_{3} Z_{W} Z_{c}}{Z}}\left(W_{\mu}^{+} c^{-}-W_{\mu}^{-} c^{+}\right)\right) \\
& +Z_{m}\left(W_{\mu}^{ \pm}\left(\sqrt{Z Y_{3} Z_{W} Z_{c}} D_{\mu} c^{\mp} \pm i g \sqrt{Z_{W}} c^{3} W_{\mu}^{\mp}\right)-Z_{c} Z_{\chi} \chi_{c}\left(\phi^{ \pm} \mp g c^{3} \bar{c}^{ \pm}\right) c^{\mp}\right) . \\
& +\chi_{3}\left(-\phi_{3} c^{3}+i g Y_{3} Z_{c} \bar{c}^{3} c^{+} c^{-}\right)
\end{aligned}
$$

The terms of zero order in $K, L$ and $\rho$ in (2.9) finally give

$$
s_{n} \Gamma_{n}=m^{2} \Sigma_{n, \rho}+\mathcal{O}\left(\Delta^{2}\right) .
$$

where we have defined $\Gamma_{n}=S+\Delta \Gamma_{n}$. (Again $S$ is the classical action, and $\Delta \Gamma_{n}$ is the divergent contribution from loop order $n$ to the source independent part of the effective action.) It implies that also $\Gamma_{n}$ is BRST invariant up to the mass terms. The most general parametrization of $\Gamma_{n}$ is then

$$
\Gamma_{n}=\Gamma_{n, Y M}+\Gamma_{n, G F}+\Gamma_{n, m}
$$

with

$$
\begin{aligned}
\Gamma_{n, Y M}= & Z S_{Y M}\left[A, W \sqrt{\frac{Z_{W}}{Z}}\right]=\int d^{4} x\left\{\frac{Z}{4}\left(\partial_{\mu} A_{\nu}-\partial_{\nu} A_{\mu}\right)^{2}\right. \\
& +\frac{i g}{2} Z_{W}\left(\partial_{\mu} A_{\nu}-\partial_{\nu} A_{\mu}\right)\left(W_{\mu}^{+} W_{\nu}^{-}-W_{\mu}^{-} W_{\nu}^{+}\right) \\
& -\frac{g^{2}}{4} \frac{Z_{W}^{2}}{Z}\left(W_{\mu}^{+} W_{\nu}^{-}-W_{\mu}^{-} W_{\nu}^{+}\right)^{2} \\
& \left.+\frac{Z_{W}}{2}\left(D_{\mu} W_{\nu}^{+}-D_{\nu} W_{\mu}^{+}\right)\left(D_{\mu} W_{\nu}^{-}-D_{\nu} W_{\mu}^{-}\right)\right\} \\
\Gamma_{n, G F}= & \int d^{4} x\left\{-\frac{\beta}{2} \phi_{3}^{2}-\phi_{3} \partial_{\mu} A_{\mu}+\partial_{\mu} \bar{c}^{3}\left(\partial_{\mu} c^{3}+i g \sqrt{\frac{Z_{W} Z_{c} Y_{3}}{Z}}\left(W_{\mu}^{+} c^{-}-W_{\mu}^{-} c^{+}\right)\right)\right. \\
& -\sqrt{\frac{Z_{W} Z_{c}}{Z Y_{3}}}\left(\phi^{+}-i g c^{3} \bar{c}^{+}\right) D_{\mu} W_{\mu}^{-}-\sqrt{\frac{Z_{W} Z_{c}}{Z Y_{3}}}\left(\phi^{-}+i g c^{3} \bar{c}^{-}\right) D_{\mu} W_{\mu}^{+} \\
& +Z_{c} D_{\mu} \bar{c}^{+} D_{\mu} c^{-}+Z_{c} D_{\mu} \bar{c}^{-} D_{\mu} c^{+} \\
& +g^{2} \frac{Z_{W} Z_{c}}{Z}\left(W_{\mu}^{+} \bar{c}^{-}-W_{\mu}^{-} \bar{c}^{+}\right)\left(W_{\mu}^{+} c^{-}-W_{\mu}^{-} c^{+}\right)
\end{aligned}
$$




$$
\begin{aligned}
& \left.-\alpha g^{2} \frac{Z_{\alpha} Z_{c}^{2}}{Z} \bar{c}^{+} c^{-} \bar{c}^{-} c^{+}-\alpha \frac{Z_{\alpha} Z_{c}}{Z Y_{3}}\left(\phi^{+}-i g c^{3} \bar{c}^{+}\right)\left(\phi^{-}+i g c^{3} \bar{c}^{-}\right)\right\} \\
\Gamma_{n, m}= & m^{2} \int d^{4} x\left\{\frac{\lambda}{2} A_{\mu} A_{\mu}+Z_{m} Z_{W} W_{\mu}^{+} W_{\mu}^{-}\right. \\
& \left.+\chi_{c} Z_{m} Z_{\chi} Z_{c}\left(\bar{c}^{+} c^{-}+\bar{c}^{-} c^{+}\right)+\chi_{3} \bar{c}^{3} c^{3}\right\}
\end{aligned}
$$

The mass terms $\lambda m^{2} A_{\mu} A_{\mu}$ and $\chi_{3} m^{2} \bar{c}^{3} c^{3}$ remain unrenormalized thanks to the identities (2.10) and (2.11), whereas the $W_{\mu}^{ \pm}$and charged ghost masses require in general independent renormalizations denoted by $Z_{m}$ and $Z_{m} Z_{\chi}$, respectively. In addition to the wave function renormalization constants $Z, Z_{W}, Z_{c}, Y_{3}$ we have introduced a gauge parameter renormalization constant $Z_{\alpha}$, whereas no independent renormalization of $\beta$ is allowed.

Note that the quantum effective action (3.9) (including the source terms $\sim K$, $L$ and $\rho$ given implicitely by eqs. (3.3) and (3.6)) is not the renormalized action, but just describes in a compact way the possible UV divergencies at loop order $n$. In order to renormalize the theory, counter terms have to be added to the bare Lagrangian such that the effective action (3.9) becomes finite. This can be achieved by the following rescaling of the fields, sources and parameters:

$$
\begin{array}{lll}
A_{\mu, R}=\sqrt{Z} A_{\mu}, & c_{R}^{3}=\frac{1}{\sqrt{Y_{3}}} c^{3}, & \bar{c}_{R}^{3}=\sqrt{Y_{3}} \bar{c}^{3}, \\
W_{\mu, R}^{ \pm}=\sqrt{Z_{W}} W_{\mu}^{ \pm}, & c_{R}^{ \pm}=\sqrt{Z_{c}} c^{ \pm}, & \bar{c}_{R}^{ \pm}=\sqrt{Z_{c}} \bar{c}^{ \pm}, \\
\phi_{R}^{3}=\frac{1}{\sqrt{Z}} \phi_{3}, & \phi_{R}^{ \pm}=\sqrt{\frac{Z_{c}}{Z Y_{3}}} \phi^{ \pm}, & \\
K_{\mu, R}^{3}=\sqrt{Y_{3}} K_{\mu}^{3}, & K_{\mu, R}^{ \pm}=\sqrt{\frac{Z Y_{3}}{Z_{W}}} K_{\mu}^{ \pm}, & \\
L_{R}^{3}=Y_{3} \sqrt{Z} L^{3}, & L_{R}^{ \pm}=\sqrt{\frac{Y_{3} Z}{Z_{c}}} L^{ \pm}, & \\
m_{R}^{2}=Z_{m} m^{2}, & \rho_{R}=Z_{m} \sqrt{Z Y_{3}} \rho, & \\
\lambda_{R}=\frac{\lambda}{Z_{m} Z}, & \chi_{c, R}=Z_{\chi} \chi_{c}, & \chi_{3, R}=\frac{\chi_{3}}{Z_{m}}, \\
g_{R}=\frac{1}{\sqrt{Z}} g, & \alpha_{R}=Z_{\alpha} \alpha, & \beta_{R}=Z \beta .
\end{array}
$$

Thus the model is perturbatively renormalisable. Note that in our convention Green functions of the renormalized fields $A_{\mu, R}$ etc. are finite (as is the quantum effective action); the wave function renormalization constants $Z$ etc. correspond to $Z^{-1}$ etc. used frequently in the literature [23]. (Our factors $Z$ describe the UV divergencies to loop order $n$ rather than the required counter terms, which are proportional to $Z^{-1}$.)

With a gauge invariant UV regulator (as dimensional regularization) the renormalization of all mass terms is multiplicative; quadratically divergent additive renormalization constants would only be required if one employs a naive UV cutoff. In 
dimensional regularization, the renormalisation constants can be chosen independent from the masses [22].

For completeness we give here the one loop expressions for all renormalization constants:

$$
\begin{aligned}
Z= & 1-g^{2} \frac{22}{3} I \\
Z_{c}= & 1-g^{2}(3-\beta) I \\
Z_{W}= & 1-g^{2}\left(\frac{22}{3}-\frac{9+\alpha}{2}-\beta\right) I \\
Y_{3}= & 1+g^{2}(3+\alpha) I \\
Z_{\alpha}= & 1-g^{2}\left(\frac{4}{3}-\alpha-\frac{3}{\alpha}\right) I \\
Z_{\chi}= & 1-g^{2}\left(\frac{4}{3}+2 \alpha+\beta-2 \chi_{c}-\frac{3}{\chi_{c}}-\frac{\alpha^{2}}{\chi_{c}}\right. \\
& \left.+\lambda\left(\frac{9+3 \alpha}{4}+\frac{3}{\chi_{c}}+\beta(\beta-1)\left(\frac{3}{2}+\frac{1}{2 \alpha}\right)\right)\right) I \\
Z_{m}= & 1-g^{2}\left(-\frac{13}{3}-\alpha+2 \chi_{c}+\lambda\left(-\frac{9+3 \alpha}{4}+\beta(1-\beta)\left(\frac{3}{2}+\frac{1}{2 \alpha}\right)\right)\right) I
\end{aligned}
$$

where $I$ denotes the ultraviolet divergent part of the logarithmically divergent integral

$$
\begin{aligned}
I=\left[\int_{\text {reg }} \frac{d^{d} p}{(2 \pi)^{d}} \frac{1}{p^{4}}\right]_{d i v .} & =\frac{1}{16 \pi^{2} \epsilon} & & \text { in } \mathrm{d}=4-\epsilon \text { dimensions } \\
& =\frac{1}{16 \pi^{2}} \ln \Lambda^{2} & & \text { in } \mathrm{d}=4 \text { with an UV cutoff } \Lambda .
\end{aligned}
$$

The classical (or renormalized) action of the model can actually be simplified considerably, if we integrate out the auxiliary fields $\phi^{ \pm}$, i.e. replace them by the solutions of their (algebraic) equations of motion. Then the classical gauge fixing part of the Lagrangian in (2.1) reads

$$
\begin{aligned}
\mathcal{L}_{G F}^{*}= & -\frac{\beta}{2} \phi_{3}^{2}-\phi_{3} \partial_{\mu} A_{\mu}+\partial_{\mu} \bar{c}^{3}\left(\partial_{\mu} c^{3}+i g\left(W_{\mu}^{+} c^{-}-W_{\mu}^{-} c^{+}\right)\right)+\frac{1}{\alpha} D_{\mu} W_{\mu}^{-} D_{\nu} W_{\nu}^{+} \\
& +D_{\mu} \bar{c}^{+} D_{\mu} c^{-}+D_{\mu} \bar{c}^{-} D_{\mu} c^{+}+g^{2}\left(W_{\mu}^{+} \bar{c}^{-}-W_{\mu}^{-} \bar{c}^{+}\right)\left(W_{\mu}^{+} c^{-}-W_{\mu}^{-} c^{+}\right) \\
& -g^{2} \alpha \bar{c}^{+} c^{-} \bar{c}^{-} c^{+}
\end{aligned}
$$

Apart from the fixing of the abelian gauge group, this is the continuum limit of the non-perturbative gauge fixing prescription proposed by Schaden [12, 7]. Now, 
in addition, one could integrate out the neutral ghosts and Lagrange multiplier $\phi_{3}$ preserving a local action (provided the determinant of the Laplacian is correctly regularised). It is interesting to note that, apart from the mass terms, the classical action is now invariant under two independent BRST symmetries:

$$
\begin{array}{ll}
s_{1} A_{\mu}=i g\left(W_{\mu}^{+} c^{-}-W_{\mu}^{-} c^{+}\right) & s_{2} A_{\mu}=\partial_{\mu} c^{3} \\
s_{1} W_{\mu}^{ \pm}=D_{\mu} c^{ \pm} & s_{2} W_{\mu}^{ \pm}=\mp i g c^{3} W_{\mu}^{ \pm} \\
s_{1} c^{ \pm}=0 & s_{2} c^{ \pm}=\mp i g c^{3} c^{ \pm} \\
s_{1} c^{3}=-i g c^{+} c^{-} & s_{2} c^{3}=0 \\
s_{1} \bar{c}^{ \pm}=\frac{1}{\alpha} D_{\mu} W_{\mu}^{ \pm} & s_{2} \bar{c}^{ \pm}=\mp i g c^{3} \bar{c}^{ \pm} \\
s_{1} \bar{c}^{3}=-\phi_{3} & s_{2} \bar{c}^{3}=-\phi_{3} \\
s_{1} \phi_{3}=0 & s_{2} \phi_{3}=0
\end{array}
$$

The second symmetry is just a particular $U(1)$ gauge transformation with gauge parameter $\xi c^{3}$ and is nilpotent. The first symmetry $s_{1}$ is not nilpotent, but $s_{1}^{2}$ gives again just a $U(1)$ gauge transformation [12, 7].

The divergent part in the effective action in the formulation without lagrange multipliers can be obtained eliminating them in (3.9). The gauge-fixing part then reads:

$$
\begin{aligned}
\Gamma_{n, G F}^{*}= & \int d^{4} x\left\{-\frac{\beta}{2} \phi_{3}^{2}-\phi_{3} \partial_{\mu} A_{\mu}+\partial_{\mu} \bar{c}^{3}\left(\partial_{\mu} c^{3}+i g \sqrt{\frac{Z_{W} Z_{c} Y_{3}}{Z}}\left(W_{\mu}^{+} c^{-}-W_{\mu}^{-} c^{+}\right)\right)\right. \\
& +Z_{c} D_{\mu} \bar{c}^{+} D_{\mu} c^{-}+Z_{c} D_{\mu} \bar{c}^{-} D_{\mu} c^{+} \\
& +g^{2} \frac{Z_{W} Z_{c}}{Z}\left(W_{\mu}^{+} \bar{c}^{-}-W_{\mu}^{-} \bar{c}^{+}\right)\left(W_{\mu}^{+} c^{-}-W_{\mu}^{-} c^{+}\right) \\
& \left.-\alpha g^{2} \frac{Z_{\alpha} Z_{c}^{2}}{Z} \bar{c}^{+} c^{-} \bar{c}^{-} c^{+}+\frac{Z_{W}}{\alpha Z_{\alpha}} D_{\mu} W_{\mu}^{+} D_{\nu} W_{\nu}^{-}\right\} .
\end{aligned}
$$

The model could be formulated without auxiliary fields from the beginning, and the result can be proved to be the same, in spite of the non-nilpotency of the first BRST variations $s_{1}$ in (3.14).

A particularly important case, which merits its own study, is where the $A_{\mu}$ and $c^{3}$ mass terms $\lambda m^{2} A_{\mu} A_{\mu}$ and $\chi_{3} m^{2} \bar{c}^{3} c^{3}$ vanish (by chosing $\lambda=\chi_{3}=0$ ). Now it is convenient to chose $\chi_{c}$, the ratio of the $W_{\mu}^{ \pm}$to $c^{ \pm}$masses, equal to the gauge parameter $\alpha$ : Instead of (2.4) we write for the $W_{\mu}^{ \pm}$and $c^{ \pm}$mass terms

$$
\mathcal{L}_{m}=m^{2}\left(W_{\mu}^{+} W_{\mu}^{-}+\alpha\left(\bar{c}^{+} c^{-}+\bar{c}^{-} c^{+}\right)\right)
$$

Let us return to the version with $\phi^{ \pm}$present: Comparing terms $\sim \phi^{ \pm}$in the gauge fixing part (2.3) of the classical Lagrangian to the term $\sim \rho$ in $(2.7)$, i.e. the BRST variation of the $W_{\mu}^{ \pm}$and $c^{ \pm}$mass terms, one finds that the classical action satisfies an additional linear identity, which remains thus unrenormalized: 


$$
\frac{\partial \Gamma}{\partial \rho}=-\int d^{4} x\left\{c^{+} \frac{\delta \Gamma}{\delta \phi^{+}}+c^{-} \frac{\delta \Gamma}{\delta \phi^{-}}\right\}
$$

The source $\rho$ is now actually redundant, and the Slavnov-Taylor identity (2.9) can be written without $\rho$ : One can replace the term $\sim \partial \Gamma / \partial \rho$ by (3.17), which corresponds to assigning a non-trivial BRST variation

$$
s \phi^{ \pm}=-m^{2} c^{ \pm}
$$

instead of $(2.5)$ to $\phi^{ \pm}$. (Now, however, the BRST variations on $\phi^{ \pm}$and the charged anti-ghosts are no longer nilpotent.) Due to the identity (3.17) the renormalization constant $Z_{m}$ of the mass term is no longer independent; now one has

$$
Z_{m}=\left(Z Y_{3}\right)^{-1}
$$

Instead of (3.9) the renormalized mass terms now read

$$
\begin{aligned}
\Gamma_{n, m} & =\int d^{4} x \frac{m^{2}}{Z Y_{3}}\left\{Z_{W} W_{\mu}^{+} W_{\mu}^{-}+\alpha Z_{\alpha} Z_{c}\left(\bar{c}^{+} c^{-}+\bar{c}^{-} c^{+}\right)\right\} \\
& =\int d^{4} x m_{R}^{2}\left\{W_{R \mu}^{+} W_{R \mu}^{-}+\alpha_{R}\left(\bar{c}_{R}^{+} c_{R}^{-}+\bar{c}_{R}^{-} c_{R}^{+}\right)\right\}
\end{aligned}
$$

Hence, after the redefinitions (3.10), the classical form (3.16) (with its particular ratio among the $W_{\mu}^{ \pm}$and $c^{ \pm}$mass terms) remains preserved by renormalisation.

Note that it is quite non-trivial that mass terms for $W_{\mu}^{ \pm}$and the charged ghosts alone allow to maintain quite simple Slavnov-Taylor identities, provided the BRST variation of the auxiliary fields $\phi^{ \pm}$is modified (non-vanishing) according to eq. (3.18).

\section{Renormalization Group Flows}

In massive theories the dependence of the Green functions on the masses can be decribed by the Callan-Symanzik (CS) equations. Likewise, in the case of more general infrared cutoff terms quadratic in the fields, the dependence on the infrared cutoff is given by the Wilsonian Exact Renormalization Group (ERG) equations. In this section we will discuss the features of these renormalization group flows in Yang-Mills theories in abelian gauges.

Let us start with the CS equations. The renormalization program, i.e. the determination of the counter terms order by order in perturbation theory, requires a renormalization prescription. In the present case of a massive theory a particularly simple renormalization prescription exists: one can chose as many independent oneparticle irreducible Green functions as there are independent counter terms, and require that they assume prescribed values at vanishing external momenta. Typically 
one will chose two point functions and their second derivatives with respect to the momentum (at vanishing momentum); these are particularly simple to evaluate. Now, in contrast to a minimal substraction scheme, the counter terms will depend in a well defined way on the masses.

Let us now assume that we have chosen a set of renormalized masses $m_{W}^{2}=m_{R}^{2}$, $m_{A}^{2}=\lambda_{R} m_{R}^{2}, m_{c^{3}}^{2}=\chi_{3 R} m_{R}^{2}$ and $m_{c^{ \pm}}^{2}=\chi_{c R} m_{R}^{2}$ with non-vanishing finite parameters $\lambda_{R}$ and $\chi_{R}$ after renormalization. All Green functions are ultraviolet finite by construction, as are the Green functions using a slightly different renormalization prescription $m_{R} \rightarrow m_{R}+\delta m_{R}$. Hence the derivatives of all Green functions with respect to $m_{R}$ are equally ultraviolet finite.

In order to derive the CS equations one starts with the derivative of the renormalized partition function with respect to the bare mass $m$. This derivative hits both the mass terms in the bare action, as well as the implicit mass dependence of all counter terms. The result can be expressed in terms of the variation of the renormalized effective action with respect to the renormalized mass. We will not rederive all corresponding steps here, which are discussed in various textbooks (see, e.g., [23]). The CS equation for the renormalized effective action then assumes the form

$$
\begin{gathered}
\left\{m_{R} \frac{\partial}{\partial m_{R}}+\beta_{g} \frac{\partial}{\partial g_{R}}+\beta_{\alpha} \frac{\partial}{\partial \alpha_{R}}+\beta_{\beta} \frac{\partial}{\partial \beta_{R}}+\beta_{\chi_{c}} \frac{\partial}{\partial \chi_{c R}}+\beta_{\chi_{3}} \frac{\partial}{\partial \chi_{3 R}}+\beta_{\lambda} \frac{\partial}{\partial \lambda_{R}}\right. \\
\left.+\sum_{i} \eta_{i} \int d^{4} x \varphi_{i}(x) \frac{\delta}{\delta \varphi_{i}(x)}\right\} \Gamma_{R}=\Delta_{m} \Gamma_{R} .
\end{gathered}
$$

Here we have introduced $\beta$ functions for the dimensionless parameters of the theory, the gauge coupling $g$, the gauge parameters $\alpha$ and $\beta$ (we hope that the reader will excuse this double use of $\beta$ ) and the mass ratios $\lambda$ and $\chi$ :

$$
\begin{gathered}
\beta_{g}=m_{R} \frac{\partial g_{R}}{\partial m_{R}} \quad \beta_{\alpha}=m_{R} \frac{\partial \alpha_{R}}{\partial m_{R}} \quad \beta_{\beta}=m_{R} \frac{\partial \beta_{R}}{\partial m_{R}} \\
\beta_{\chi_{c}}=m_{R} \frac{\partial \chi_{c R}}{\partial m_{R}} \quad \beta_{\chi_{3}}=m_{R} \frac{\partial \chi_{3 R}}{\partial m_{R}} \quad \beta_{\lambda}=m_{R} \frac{\partial \lambda_{R}}{\partial m_{R}} .
\end{gathered}
$$

$\eta_{i}$ denote the various anomalous dimensions:

$$
\eta_{i}=\frac{m_{R}}{2} \frac{\partial \log \left(Z_{i}\right)}{\partial m_{R}}
$$

The right hand side $\Delta_{m} \Gamma_{R}$ of eq. (4.1) consists of the sum of (bare) mass terms and one loop diagrams with mass insertions:

$$
\Delta_{m} \Gamma_{R}=\frac{m_{R}^{2} \gamma_{m}}{Z_{m}} \int d^{d} x\left\{Z_{W}^{-1}\left[W_{\mu, R}^{+} W_{\mu, R}^{-}+\frac{1}{2} \operatorname{Tr}\left[\Gamma^{(2)}\right]_{W_{\mu, R}^{+}, W_{\nu, R}^{-}}^{-1}\right]\right.
$$




$$
\begin{aligned}
& +\frac{\lambda_{R} Z_{m}}{2} Z_{\lambda}^{-1}\left[A_{\mu, R} A_{\mu, R}+\operatorname{Tr}\left[\Gamma^{(2)}\right]_{A_{\mu, R}, A_{\nu, R}}^{-1}\right] \\
& +\chi_{c R} Z_{\chi}^{-1} Z_{c}^{-1}\left[\bar{c}_{R}^{ \pm} c_{R}^{\mp}-\operatorname{Tr}\left[\Gamma^{(2)}\right]_{c_{R}^{ \pm}, \bar{c}_{R}^{\mp}}^{-1}\right. \\
& \left.+\chi_{3 R} Z_{c}^{-1}\left[\bar{c}_{R}^{3} c_{R}^{3}-\operatorname{Tr}\left[\Gamma^{(2)}\right]_{c_{R}^{3}, \bar{c}_{R}^{3}}^{-1}\right]\right\}
\end{aligned}
$$

where we have defined

$$
\gamma_{m}=\frac{Z_{m}}{m_{R}} \frac{\partial m^{2}}{\partial m_{R}}=2-m_{R} \frac{\partial \log \left(Z_{m}\right)}{\partial m_{R}} .
$$

The expressions $\left[\Gamma^{(2)}\right]_{\varphi^{a}, \varphi^{b}}^{-1}$ denote the one loop diagrams which correspond to the $\varphi^{a}, \varphi^{b}$ propagators (in an arbitrary background) at coincident points.

Although the individual terms in (4.4) are not separately ultraviolet finite, renormalizability ensures that their sums - as well as all $\beta$ functions and anomalous dimensions $\eta_{i}$ in (4.1) - are finite in the limit of an infinite ultraviolet cutoff $\Lambda$ [23].

The use of the CS equation (4.1) consists in the study of the behaviour of Green functions at large Euclidean momenta $p^{2}$ : After having removed an ultraviolet cutoff $\Lambda$, dimensionless Green functions can only depend on the ratio $p^{2} / m_{R}^{2}$ for dimensional reasons. Furthermore, for $p^{2} / m_{R}^{2} \gg 1$, the right hand side of (4.1) becomes negligeable [23]. Writing $\partial / \partial m_{R}^{2}=-\partial / \partial p^{2}$ the desired renormalization group equation describing the $p^{2}$ dependence is then easily obtained.

In the case of Yang-Mills theories in abelian gauges one finds, using our results of the preceeding section and in [7, 19, 24], that several $\beta$ functions are related to the anomalous dimension $\eta_{A}$ of $A_{\mu}$ :

$$
\begin{aligned}
& \beta_{g}=-g_{R} \eta_{A} \\
& \beta_{\beta}=-2 \beta_{R} \eta_{A} \\
& \beta_{\lambda}=-\lambda_{R}\left(2-\gamma_{m}+2 \eta_{A}\right)
\end{aligned}
$$

(Note that $\beta_{R}$ on the right hand side of (4.6b) denotes the renormalized gauge parameter.)

Equation (4.6a) implies that $\beta_{g}$ can be computed from the $A_{\mu}$ propagator alone [24]. Now recall eqs. (4.2), i.e. the fact that in the context of the CS equations the $\beta$ functions are given by the derivatives of the counter terms with respect to the masses. One finds that

a) to one loop order, $A_{\mu}$ propagators do not contribute to the renormalization of the $A_{\mu}$ propagator itself (i.e. $\eta_{A}$ ), and

b) even to two loop order the renormalization of the $A_{\mu}$ propagator is infrared finite for $m_{A} \rightarrow 0$ or $\lambda \rightarrow 0$.

This allows to obtain the two-loop $\beta$ function of Yang-Mills theories in the particularly simple approach discussed near the end of the preceeding section: It is 
possible to choose $m_{A}=m_{c^{3}}=0\left(\right.$ or $\left.\lambda=\chi_{3}=0\right)$ and $m_{c^{ \pm}}^{2}=\alpha m_{W}^{2} \quad\left(\right.$ or $\left.\chi_{c}=\alpha\right)$, and thus to invoke the particularily simple version of the Slavnov-Taylor identities obtained by the use of eq. (3.17). Up to the universal two loop order $\beta_{g}$ is then obtained by the variation of $Z_{A}$ with respect to $m_{W}$ and $m_{c^{ \pm}}$only.

Eq. (4.6c) shows that $m_{A}=0$ (or $\lambda=0$ ) is stable under the renormalization group flow, and furthermore eq. (4.5) together with (3.19) implies that $\gamma_{m}$ is now given by

$$
\gamma_{m}=2\left(1+\eta_{A}+\eta_{\bar{c}^{3}}\right)
$$

However, for $m_{A}=0$ not all Green functions are infrared finite at vanishing external momenta. Already the one loop contributions to the four point functions with four $W_{\mu}^{ \pm}$bosons (or two $W_{\mu}^{ \pm}$bosons and two charged ghosts, or four charged ghosts) involving two $W_{\mu}^{ \pm} W_{\mu}^{\mp} A_{\mu} A_{\mu}$ vertices are infrared divergent in this case. Hence non-vanishing external momenta have to be chosen at the renormalization points for at least some counter terms, and not all $\beta$ functions and anomalous dimensions are given just by the variation with respect to $m_{W}$ (and $m_{c}$ ) only.

The ERG equations [16, 17] describe equally the variation of the Green functions with respect to an infrared cutoff, which is introduced in the form of additional terms quadratic in the fields to the bare action. In the case of Yang-Mill theories modified Slavnov-Taylor identities have to be imposed [17]; the corresponding modifications correspond to the term $\sim \partial \Gamma / \partial \rho$ in our eq. (2.9). (In the context of ERG equations a corresponding source $\rho$ could, in principle, also be introduced; this would not particularly simplify, however, a parametrization of the effective action which satisfies the modified Slavnov-Taylor identities.)

Various forms of such cutoff terms have been discussed in the literature [16, 25], and mass terms as discussed here would of course be particularly simple. In the context of non-abelian Yang-Mills theories they have been proposed in [26], but they lead to conceptional problems.

First, a mass term as infrared cutoff in the ERG context corresponds to a bare mass. Whereas the renormalizability of the theory implies that derivatives of the Green function with respect to the renormalized mass are ultraviolet finite (cf. the CS equations), ultraviolet finiteness of derivatives of Green function with respect to the bare mass will generically not be given. For this reason of ultraviolet finiteness of the ERG equations the infrared cutoff terms have to have a non-trivial momentum dependence in general; notably a simple mass term for $W_{\mu}^{ \pm}$and the charged ghosts only (with its simple modifications of the Slavnov-Taylor identities) can, unfortunately, not be used to define an ultraviolet finite exact renormalization group flow. Second, as discussed above, $m_{W}$ alone would not serve as an infrared regulator for all Green functions; the remaining infrared divergences reduce to the ones of QED with matter which are, however, much easier to deal with.

Let us add another twist to the difference between bare and renormalized mass terms. Clearly, in order to recover the partition function of QCD, we have to consider the limit of vanishing bare mass terms. Is it possible to maintain non-vanishing 
renormalized masses in this limit, and how can these agree with the Slavnov-Taylor identities? This question is particularly acute in view of the finite $W$ masses observed on the lattice [3].

First we note that our parametrization (3.9) of the quantum effective action to $n$ loop order is not only useful in order to constrain the corresponding ultraviolet divergences: After having renormalized the theory, we can also ask what is the most general form (consistent with the Slavnov-Taylor identities) of the perturbatively relevant terms in the full quantum effective action. Again this is given by the parametrization (3.9); now, however, the renormalization constants $Z_{i}$ denote simply the behaviour of the corresponding Green functions in the limit of vanishing external momenta: By definition, the coefficient of each term (specified by its field content) in the effective action denotes the one particle irreducible Green function with the corresponding fields as (amputated) external lines. These Green functions depend on the external momenta, and will approach the form of the bare action in the limit of large Euclidean non-exceptional external momenta. In the limit of vanishing external momenta, these Green functions can well diverge in a massless theory.

Within this new interpretation of the parametrization (3.9) of the quantum effective action the parameters $g, m, \lambda$ and $\chi$ are finite (renormalized) quantities, and the coefficients $Z, Y$ are ultraviolet finite numbers which possibly diverge for $m \rightarrow 0$. Standard Yang-Mills theory is obtained in the limit $m \rightarrow 0$, since in this limit the Slavnov-Taylor identities (2.9) turn into the standard massless identities.

Let us now concentrate on the case $\lambda, \chi_{3}=0$, where the mass terms for $A_{\mu}$ and the neutral ghosts have already been switched off; then we are left to consider the mass terms $m^{2} Z_{m}\left(Z_{W} W_{\mu}^{+} W_{\mu}^{-}+\chi_{c} Z_{\chi} Z_{c}\left(\bar{c}^{+} c^{-}+\bar{c}^{-} c^{+}\right)\right)$in (3.9) in the limit $m^{2} \rightarrow 0$. From (3.19) we find that they could actually remain finite in this limit, provided the product $Z Y_{3}$ vanishes sufficiently rapidly for $m^{2} \rightarrow 0$. This would allow for a massive behaviour of the $W$ propagator, maintaining the standard form of the Slavnov-Taylor identities (together with standard BRST variations for $\phi^{ \pm}$); however, one carefully has to study the effects of $Z Y_{3} \rightarrow 0$ in all the other terms of the action:

a) For $Z \rightarrow 0$ the Slavnov-Taylor identities require the renormalized coupling to diverge for vanishing external momenta. On the one hand $Z \rightarrow 0$, in $S U(N)$ covariant gauges, is believed to be a signal for confinement 18], on the other hand lattice results 27] (again in $S U(N)$ covariant gauges) indicate that effective vertices remain finite in this limit. It is not clear which of these results holds in abelian gauges considered here; in fact the abelian model for confinement proposed in [10] would correspond to the case $Z \rightarrow 0$.

b) $Y_{3} \rightarrow 0$ seems to be a possibility specific to abelian gauges: After the elimination of $\phi^{ \pm}$by its equations of motion, one finds from eq. (3.15) that the effective action is well behaved in this limit. (Actually, if one includes the counter terms in the BRST variations (3.14) after the elimination of $\phi^{ \pm}$, one finds that all variations $s_{1}$ are proportional to $Y_{3}$ or $\sqrt{Y_{3}}$. Hence in the limit $Y_{3} \rightarrow 0$ the BRST symmetries reduce to the abelian ones, which explains the possiblity to have finite $W$ masses 
consistent with standard Slavnov-Taylor identities, without infrared divergencies.)

Note that, whereas $Z \rightarrow 0$ has an effect on the gluonic vertices of the theory, $Y_{3}$ appears only in the gauge fixing part (and mass terms) of the action; hence it resembles more to a gauge parameter. Hence, after renormalization, one could envisage to tune both $m \rightarrow 0$ and $Y_{3} \rightarrow 0$ with $m^{2} / Y_{3}$ finite and to define a renormalizable massive theory with standard Slavnov-Taylor identities this way; (perturbative) unitarity of this model remains, however, to be investigated.

\section{Conclusions}

We have constructed a massive generalisation of SU(2)-Yang-Mills theory in an abelian gauge. Its renormalizability can be shown thanks to a simple generalisation of the Slavnov-Taylor identities. The possibility to renormalise a (pure) Yang-Mills theory for fixed finite masses (which can be chosen, due to asymptotic freedom, such that the theory is completely perturbative) allows to separate the renormalisation process from infrared phenomena.

After completion of the renormalization program one will typically be interested in removing the "artificial" mass terms. We have discussed, in which cases this procedure defines consistent renormalization group flows. Such renormalization group flows can actually be used directly as tools in order to probe the infrared behaviour of Yang-Mills theories.

It is probable that confinement (via monopole condensation) is more easily describable in abelian gauges. Then the massive version in such gauges will be particularly useful. In the abelian gauge we have to distinguish between the abelian "diagonal" and the "off-diagonal" gluons, and we carefully discussed the different properties of the different mass terms. We cannot identify these masses "for symplicity" without spoiling the renormalisability. The possibility to maintain finite renormalized masses for the (off-diagonal) $W$ gauge bosons for vanishing bare mass terms seems to be promising, and may help to understand some aspects of the infrared limit of Yang-Mills theories.

\section{Acknowledgement}

We would like to thank L. Baulieu for helpful discussions.

\section{References}


[1] J. M. Cornwall, D. N. Levin, G. Tiktopoulos, Phys. Rev. D10 (1974) 1145.

For a review see J. Horejsi, Introduction to Electroweak Unification. Standard Model from Tree Unitarity, Word Scientific (New York 1993).

[2] C. Bernard et al, Phys. Rev. D49 (1994) 1585;

P. Marnzoni, G. Martinelli, N. Stella, Nucl. Phys B455 (1995) 339;

P. Marenzoni et al., Phys. Lett. B318 (1993) 511;

D. B. Leinweber et al., Phys. Rev. D58 (1998) 031501, and Phys. Rev. D60 (1999) 094507, Erratum ibid D61 (2000) 079901;

A. Nakamura, S. Sakai, Prog. Theor. Phys. Suppl. 131 (1998) 585;

D. Becirevic et al., Nucl. Phys. Proc. Suppl. 83 (2000) 159, and Phys. Rev. D61 (2000) 114508.

[3] K. Amemiya, H. Suganuma, Phys. Rev. D60 (1999) 114509, and Nucl. Phys. B (Proc. Suppl.) 83-84 (2000) 419 and 547.

[4] G. t'Hooft, Nucl. Phys. B 190 [FS3] (1981) 455.

[5] A. Kronfeld, M. Laursen, G. Schierholz, U. J. Wiese, Phys. Lett. B198 (1987) 516 ;

T. Suzuki, I. Yotsuyanagi, Phys Rev. D25 (1990) 4257;

A. Di Giacomo, hep-lat/9603029 and hep-lat/9802008;

M. Polikarpov, hep-lat/9609020;

M. Chernodub and M. Polikarpov, hep-th/9710205;

G. Bali, hep-ph/980935.

[6] S. Mandestam, Phys. Lett. B53 (1975) 476, and Phys. Rep. C 23 (1976) 245;

G. t'Hooft, Nucl. Phys. B190 (1981) 455.

[7] M. Schaden, hep-th/9909011 v3.

[8] K.-I. Kondo, Phys. Lett. B514 (2001) 335;

K.-I. Kondo, T. Shinohara, Phys. Lett. B491 (2000) 263.

[9] A. M. Polyakov, Gauge Fields and Strings, Harwood Academic Publishers (Chur 1987).

[10] U. Ellwanger, N. Wschebor, Phys. Lett. B517 (2001) 462, and JHEP 0110 (2001) 023.

[11] V. N. Gribov, Nucl. Phys. B139 (1978) 1.

[12] M. Schaden, Phys. Rev. D59 (1999) 014508.

[13] G. Curci and R. Ferrari, Nuovo Cim. A32 (1976) 1, ibid A35 (1976) 1;

G. Curci and E. d'Emilio, Phys. Lett. B83 (1979) 199;

I. Ojima, Z. Phys. C13 (1982) 173; 
R. Delbourgo, S. Twisk and G. Thompson, Int. J. Mod. Phys. A3 (1988) 435;

A. Basi, N. Maggiore, Mod. Phys. Lett. A11 (1996) 1665.

[14] J. de Boer, K. Skenderis, P. van Nieuwenhuizen, A. Waldron, Phys. Lett. B367 (1996) 175-182.

[15] C. G. Callan, Phys. Rev. D2 (1970) 1541;

K. Symanzik, Commun. Math. Phys. 18 (1970) 227.

[16] K. Wilson and I. Kogut, Phys. Rep. 12 (1974) 15;

F. Wegner, in Phase Transitions and Critical Phenomena, Vol 6, Academic Press (New York 1975);

J. Polchinski, Nucl. Phys. B231 (1984) 269;

G. Keller, C. Kopper, Phys. Lett. B273 (1991) 323;

C. Wetterich, Phys. Lett. B301 (1993) 90;

U. Ellwanger, Z. Phys. C58 (1993) 619;

M. Bonini, M. D'Attanasio, G. Marchesini, Nucl. Phys. B409 (1993) 441;

C. Wetterich, M. Reuter, Phys Lett. B334 (1994) 412;

T. Morris, Int. J. Mod. Phys. A9 (1994) 2411;

M. D'Attanasio, T. Morris, Phys. Lett. B378 (1996) 213;

G. Keller, C. Kopper, M. Salmhofer, Helv. Phys. Acta 65 (1997) 32;

For a review, see J. Berges, N. Tetradis, C. Wetterich, hep-ph/0005122.

[17] C. Becchi, in Elementary Particles, Field Theory and Statistical Mechanics, M. Bonini, G. Marchesini and E. Onofri eds., Parma University, 1993;

M. Reuter, C. Wetterich, Nucl. Phys. B417 (1994) 181;

M. Bonini, M. D’Attanasio, G. Marchesini, Nucl. Phys. B418 (1994) 81, and Nucl. Phys. B421 (1994) 429;

U. Ellwanger, Phys. Lett B335 (1994) 364;

M. D'Attanasio, T.R. Morris, Phys. Lett. B378 (1996) 213;

U. Ellwanger, M. Hirsch, A. Weber, Z. Phys. C 69 (1996) 687.

[18] R. Oehme, Phys. Rev. D42 (1990) 4209, and Phys. Lett. B252 (1990) 641;

K. Nishijima, Int. J. Mod. Phys. A9 (1994) 3799, and Czech. J. Phys. 46 (1996) 1 .

[19] H. Min, T. Lee and P. Y. Pac, Phys. Rev. D32 (1985) 440.

[20] A. R. Fazio, V.E.R. Lemes, M.S. Sarandy, and S.P. Sorella, Phys. Rev. D64 (2001) 085003.

[21] I. V. Tyutin, Lebedev preprint, FIAN 39 (in Russian) (1975);

C. Becchi, A. Rouet and S. Stora, Commun. Math. Phys. 42, (1975) 127, and Ann. Phys. 98, (1976) 287.

[22] S. Weinberg, Phys. Rev. D8 (1973) 3497. 
[23] J. Zinn-Justin, Quantum Fields Theory and Critical Phenomena, Oxford University Press (Oxford 1989). C. Itzykson, J. B. Zuber, Quantum Field Theory, McGraw-Hill (New York 1980).

[24] M. Quandt, H. Reinhardt, Phys. Lett. B424 (1998) 115, and Int. J. Mod. Phys. A13 (1998) 4049.

[25] D. F. Litim, Phys. Rev. D64 (2001) 105007.

[26] M. Simionato, Int. J. Mod. Phys. A15 (2000) 2121, Int. J. Mod. Phys. A15 (2000) 2153, and Int. J. Mod. Phys. A15 (2000) 4811.

[27] P. Boucaud, J.P. Leroy, J. Micheli, O. Pene, C. Roiesnel, JHEP 004 (1998) 9812. 